\title{
EIN SPIEL MIT STEREOTYPEN? ZU MATTHIAS NAWRATS ROMAN DIE VIELEN TODE UNSERES OPAS JUREK (2015)
}

\begin{abstract}
Playing with stereotypes? In regard to Matthias Nawrats's novel 'Die vielen Tode unseres Opas Jurek' (2015)

Matthias Nawrat, born in 1979 in Opole, has been living in Germany since 1989. In his latest novel Die vielen Tode unseres Opas Jurek (2015) the author returns to the country of his birth in order to recount one century of the Polish as well as of the Polish-German history. The ghastly-comical family-story uses mobile parts of stage scenery from a picaresque novel - in the centre stands a man (grandfather Jurek) who, in spite of all the atrocities of the 20th century, won't let others prevent him from leading a joyful life. While staging the story of a struggling artist, he refers back to stereotypes, obviously with the intention of strenghtening the existing models. This contribution poses a question if the game has been successful.
\end{abstract}

KEY WORDS: Matthias Nawrat, stereotype, game

Bekanntlich entstand der Begriff ,Stereotyp“ in seiner primären, rein auf die Technologie bezogenen Form um das Jahr 1798. Sein Schöpfer, der französische Buchdrucker Firmin Didot, setzte den Terminus aus den griechischen Wörtern

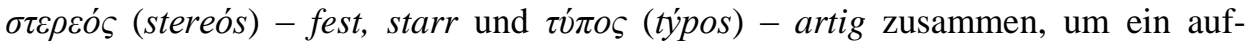
wendiges Buchdruckverfahren zu bezeichnen: Eine komplette Buchdruckplatte wird von den aus beweglichen Lettern gesetzten Druckseiten durch Abformen über eine Matrize und deren finalen Abguss in eine Metalllegierung erstellt (Pająk-Patkowska 2011, S. 5). Eben jene Beschaffenheit einer Matrize, die sich durch Rigidität auszeichnet, durch eine ein für alle Mal geprägte und daher nicht mehr korrigierbare Form, veranlasste den Journalisten Walter Lippmann, dem Wort eine Neubedeutung zu geben. Die semantische Neuausrichtung fand nicht nur Eingang in die Sozialpsychologie, sie schlug sich auch in einer Einbettung in allgemeinen Vorstellungen nieder. Wie Krystyna Mihułka bemerkt, geht Lippmann in seinem 1922 in New York erstveröffentlichten Buch Public Opinion von einer plakativen Vorstellung 
aus: „the world outside and the pictures in our heads“ - um daran anknüpfend seine Kritik an der selektiven Wirklichkeitswahrnehmung durch Massenmedien seiner Zeit zu üben. Bis heute gilt Lippmanns Buch in solchen Disziplinen wie Kommunikations-, Politik-, oder Sozialwissenschaft als Basis für Debatten über das Wesen des Stereotyp-Begriffs (Mihułka 2010, S. 78).

Inzwischen versteht man unter diesem Begriff die im Laufe des Sozialisierungsprozesses angehäuften ,Bilder im Kopf ${ }^{\prime}$. Laut zahlreichen Definitionen dienen sie ihren Nutzern in erster Linie zur (Schein)definition der sie umgebenden Welt und damit auch als gängige Instrumente zur „Verteidigung der eigenen Position“ (Mihułka 2010, S. 78) - sie zielen auf eine Distanzierung bzw. Separierung von ,Fremdem' ab, legen also fest, was abgewehrt bzw. integriert werden sollte, und liefern somit ein ,geordnetes, mehr oder minder beständiges Weltbild“ (Mihułka 2010, S. 79). In ihrer Funktion als simplifizierende Urteile, die die Komplexität der Welt auf einfache Muster reduzieren, erfüllen Stereotype ihre Rolle in der ethnischen, kulturellen, historischen, politischen und nicht zuletzt auch sozialen Wirklichkeit der jeweiligen Kultur- und Sprachgemeinschaft. Sie können Träger von positiven und/oder negativen Vorstellungen bzw. Urteilen/Vorurteilen sein.

Stereotype sind keinesfalls absichtliche Lügen. Allerdings sind sie - da mit einem relativen Quantum an Wahrheitsgehalt behaftet - meistens nichts anderes als ein Resultat radikaler Vereinfachung komplexer Sachverhalte. In selteneren Fällen stellen sie das Ergebnis einer vorschnellen Generalisierung eigener, bewusst zweckgefärbt ausgewerteter Einzelerfahrungen dar (Popovic 1998). Da sie trotz exzessiven Gebrauchs kaum Verschleißerscheinungen aufweisen, sind sie schnell zur Hand, wenn es darum geht, die Welt in der Gesamtheit ihrer Phänomene auf eine schematisierte, griffige und schnell abrufbare Art und Weise zu klassifizieren. Im Vergleich zu den - allein schon ihrem erkenntnisorientierten Wesen nach - formbaren, weil dynamisch funktionierenden Begriffen, die aus einer reflektierenden Auseinandersetzung mit kontinuierlich neu erworbenem Wissen hervorgehen, zeichnen sich Stereotype durch eine weitgehende Resistenz aus - weder neu erworbenes Wissen noch persönliche Erfahrung führen zu ihrer Falsifizierung. Besonders interessant scheint in diesem Kontext das Phänomen der „Stereotype der langen Dauer“ zu sein. Ihre Beständigkeit, die sich jedem Versuch einer objektivierenden Beurteilung verweigert, liege nämlich, so Hubert Orłowski, nicht etwa allein darin, ,dass sie lange und in praxi ununterbrochen funktionieren, sondern, dass sie in jedem geeigneten Moment wieder zum Leben berufen werden können“. (Orłowski 2005, S. 18) Beide Eigenschaften - sowohl der lange Fortbestand als auch die Möglichkeit jederzeitiger Reaktivierung - wirken dann umso nachhaltiger, wenn sich das jeweilige, oft bis hin zum Gegenstand einer bloßen Taxonomie abgewertete Objekt der jeweiligen Stereotypisierung mehrere „Jahrhunderte lang auf der Respekt-Despekt-Skala notorisch am Ende der Skala befindet" (Orłowski 2005, S. 17-18). 
Ein solches auf stereotypen Matrizen fußendes, stets aktivierbares Wahrnehmungsspiel stellt geradezu beispielhaft die Beschäftigung der öffentlichen Meinung Westeuropas mit zivilisatorischen Aspekten des Ost-West-Gefälles dar. Ungeachtet der meist nur an der gesellschaftlichen Oberfläche greifenden Darstellungs- und Verhaltensmodi, im Sinne der politischen Korrektheit etwa, lässt sich der nach wie vor argwöhnisch gen ,Osten` gerichtete Blick von einem durch Stereotype genährten Zerrbild angeblich desolater Zustände in Ostmitteleuropa leiten. László Végel (2008) geht in seinem ironischen, die Grenzen des Schablonendenkens bewusst ausreizenden ,West-Check ${ }^{e}$ der mitteleuropäischen Seele eindringlich auf die in der westeuropäischen Optik des Ostens omnipotenten Klischees ein:

Der mittelosteuropäische Mensch wäre gern anderswo auf die Welt gekommen, so tröstet er sich mit dem nostalgischen Gedanken an Europa. Weil er kein Westeuropäer ist, verflucht er sein Schicksal. Er leidet an ewigem Minderwertigkeitskomplex, sein tiefer Wunsch, nicht hier zu sein, hindert ihn, seinen Nachbarn kennen zu lernen. Er erfährt ihn nur dann, wenn er zufällig irgendwo weit weg in der Fremde gelandet ist. Das ist verzeihlich, denn er wird auch mit seinem Selbst erst in einer solchen Situation konfrontiert. So verändert es nichts, wenn hier alle ein doppeltes Leben führen, jeder muss einmal sein Zuhause verlassen, um seine Identität zu erkennen. (S. 51)

Die bereits angedeutete Ost-West-Despekt-Wahrnehmung findet ihre auffällige Variante an einer - was die Fülle und Hartnäckigkeit von gängigen Stereotypen anbetrifft - besonderen Schnittstelle, dort nämlich, wo es zur Gegenüberstellung der deutschen und der polnischen Kultur- und Lebensart sowie der beiden Mentalitäten kommt. Wie Hubert Orłowski feststellt, geht es um „die im Hintergrund funktionierenden Stereotype der langen Dauer, um den Zusammenstoß zweier ,Sonderwege“, des preußisch-deutschen und des in seiner historischen Bewegung erstarrten polnischen“. (Orłowski 2005, S. 113) In der auf überlieferte Vorstellungen fixierten, dichotomischen Abgrenzung des deutschen und des polnischen Nationalcharakters werden die Stereotype eher intensiviert als destruiert. Streitsucht, übertriebener Nationalstolz, anarchistische Freiheitsliebe, Armut und Schmutz, Chaos, Verwirrung, schließlich die unter dem Begriff ,polnische Wirtschaft' subsummierte Unordnung mit der darin inbegriffenen Gesamtheit aller Improvisierungsbemühungen versus (deutsche) Ordnungsliebe und Zielstrebigkeit, Effizienz und Sparsamkeit, Rechtschaffenheit und Gesetzestreue - dies seien westwärts der deutsch-polnischen Grenze immer noch die vorherrschenden Auffassungen in puncto polnischer Habitus: „Der deutsche Polendiskurs verfügte und verfügt wohl immer noch über eine außerordentliche moralisch-zivilisatorisch begründete Macht, sich über Polen mit nationalem Respekt bzw. despektierlich zu äußern.“ (Orłowski 2005, S. 154)

Im Grunde bleibt die Frage, ob überhaupt (und wenn ja, dann inwiefern erfolgreich) mit den Mitteln/Waffen der Literatur gegen die Hartnäckigkeit solcher jahrhundertelangen Stereotype vorgegangen werden kann, so gut wie unbeantwortet. Gewisse Erfolgschancen verspricht dem angehenden Forscher die Lektüre einer von 
anerkannten Fachgrößen verfassten, profund-wissenschaftlichen Abhandlung. Der wissbegierige Laie wiederum wird zu einem auf solide Recherchen gestützten Sachbuch greifen. In beiden Fällen stellt das zur Entstehung des nichtfiktionalen Werks herangezogene historisch-statistische, ökonomische und soziologische Instrumentarium einen Betrachtungswechsel in Aussicht. Im Bereich der fiktionalen Literatur erweist sich die Bewerkstelligung dieser Aufgabe jedoch als heikel. Der fiktionale Text ist meistens an einen künstlerischen Anspruch gekoppelt, was ihn im Dienste sachlicher Aufklärung oder kritischer Revision einer vorgefundenen Bewusstseinslage kaum verwendbar macht. Statt aufzuklären, fungiert er letztendlich nicht selten als affirmativer oder gar pervertierter Darstellungsmodus, der die althergebrachten Bilder, Symbole, Mythen und Vorstellungen des Anderen seinerseits zu verfestigen droht.

Will man die Aufmerksamkeit des deutschsprachigen Lesers auf die Realität Ostmitteleuropas - in diesem Falle Polens - sowie auf die durch gesellschaftlichhistorische Prozesse geformte Mentalität seiner Bewohner lenken und ihn zu einer Auseinandersetzung anregen, die - wenn auch gewiss nie erschöpfend, so doch wenigstens annähernd - die Angelpunkte der Gesamtproblematik erfassen könnte, dann sollte dies vielleicht am effizientesten über Texte eines ostmitteleuropastämmigen, deutschsprachigen Schriftstellers geschehen. Dieser schildert die gesamte Problematik aus dem Reservoir eigener Erfahrung heraus: Da er das jeweilige Biotop als Insider gut kennt, erzählt er seine Geschichte sozusagen aus erster Hand. Damit fällt der Zwang weg, die jeweilige Realität erst mühsam aus der Perspektive eines Fremden $\mathrm{zu}$ entschlüsseln. Das Insiderwissen des jeweiligen Autors setzt allerdings keine Totalität seiner Darstellung voraus - schließlich ist der Text für Menschen ohne profunde Kenntnis des polnischen Habitus bestimmt. Die potentiellen Leser sind also gerade nicht in die Gesamtheit aller Prozesse und Fakten involviert, mehr noch: Sie streben dieses Involviert-Sein auch gar nicht erst an. Das literarische Abbild der Realität erhebt zumeist keinen Anspruch auf Vollständigkeit und strebt so gut wie nie eine Revision der gängigen Vorstellungen an, vielmehr bestätigt es diese. Für den Leser bedeutet es, dass er bei der Lektüre gewisse Verallgemeinerungen in Kauf nehmen muss. Sie werden durchaus akzeptiert, da sie ein festes Weltbild von den Nachbarn bestätigen und nebenbei - allein schon durch die Person des Autors - den Schein der vollen Authentizität wahren helfen. Im Endeffekt hat man bei einer narrativen Geste dieser Art oft mit einer leserfreundlichen Bestätigung bestehender Klischees zu tun und ,,begegnet [...] einer vorgefertigten, grundsätzlich negativ gefärbten Folie. Und man beginnt nun im Prozess der habituellen Selbstreflektion dieses Bildnis zu übernehmen und den fremden Glanz (rück)auszustrahlen“. (Orłowski 1996, S. 400)

Der Roman Die vielen Tode unseres Opas Jurek (Nawrat 2015) ist das dritte Buch des 1979 in Opole geborenen, als knapp Zehnjähriger mit seinen Eltern nach Bamberg ausgewanderten Autors Matthias Nawrat. Seinem 2012 erschienenen De- 
büt Wir zwei allein, einer detaillierten Schilderung einer singulären ,vegetarischen Amour fou in Freiburg“" (Halter 2012) folgte 2014 Unternehmer, laut Björn Hayer eine „höchst aktuelle Karikatur unserer Gesellschaft“ (Hayer 2014). Das dritte Werk Nawrats stellt den Versuch einer literarischen Rückkehr in das vertraute polnische Urheimatgefilde im niederschlesischen Opole dar. Freilich ist es keine Nostalgiereise um der Nostalgie willen. Den traurigen Anlass für den Opole-Besuch des vor Jahren mit den Eltern in die BRD ausgewanderten Enkels liefert das anstehende Begräbnis von Opa Jurek, dem Großvater mütterlicherseits. Es handelt sich um eine plakative Trauerfeier, bei der geradezu selbstverständlich ein „Gedicht des großen polnischen Poeten Adam Mickiewicz mit dem Titel ,Im Stammbuch““ (Nawrat 2015, S. 8) aufgesagt werden musste, und in deren Verlauf der Pfarrer in wahrer Showmastermanier - erzpolnisch - ,in seinem weißen Gewand aussah wie Johannes Paul II. höchstpersönlich, klackernd mit seiner Lampe aus Silber an einer Silberkette [wedelte], und der Weihrauch schwebte über alle Köpfe“. (Nawrat 2015, S. 7-8)

Der Roman beginnt also in der Gegenwart, um bald eine Fülle von verzweigten Rückgriffen in die Vergangenheit darzubieten. Für Tomasz Kurianowicz seien die „ironischen, schier unfassbaren Wendungen in dieser halb erdichteten, halb faktisch grundierten Biographie [...] zugleich die ironischen Brechungen Polens" (Kurianowicz 2015). Aus weitgefächerten Bruchstückdarstellungen des Vergangen-Gedachten spinnt Nawrat eine effektvoll arrangierte Story, in der die große Geschichte das eigentliche Geschehen immer wieder zu beschleunigen scheint. Als Hauptbezugsfigur verkörpert Opa Jurek in seiner Urwüchsigkeit das Klischee des schlauen Polen, der sich niemals und von niemandem unterkriegen lässt - dass es dabei nicht immer ganz gesetzeskonform zugeht, versteht sich fast von selbst. In dem aus insgesamt 32 grotesken Episoden zusammengefügten Familienepos werden die Biographien der allernächsten Familienmitglieder sowohl mütterlicher- als auch väterlicherseits nahtlos in über 60 Jahre polnischer Geschichte eingereiht. Die Erzählinstanz ist dabei eine der besonderen Art - an die Stelle des Ich-Erzählers tritt ein Wir-Erzähler. Nico Bleugte expliziert dazu: „Ein nicht genauer bestimmtes ,Wir‘ gibt die Perspektive vor, ein Wir, bei dem es sich um ein Geschwisterpaar handeln könnte oder um mehrere Brüder und Schwestern im Chor, die aus der Sicht der Enkel erzählen." (Bleutge 2015) Onkel, Tanten, Großeltern und - allen voran - die Eltern stehen aber als Bezugspersonen fast durchgehend im Vordergrund der narrativen Bewegung, denn die Wir-Erzähler Nawrats platzieren die Einzelepisoden ihrer Geschichte in den Bereich der familiären Kleinhistorie.

Der Zustand einer allgemeinen Vertrautheit und das Gemeinschaftsgefühl erwachsen aus dem engen Zusammenspiel verwandtschaftlicher Beziehungen, denen eine Schutzfunktion innewohnt: Die familiären Bindungen ersetzten mit Erfolg, allem nationalen Unglück trotzend, die fehlenden Strukturen eines Staates, auf den in den periodisch auftretenden Umbrüchen erfahrungsgemäß kaum Verlass sein konnte: 
Die Menschen in Polen zum Beispiel könnten auf viele schwere Zeiten zurückschauen. Aber nicht einmal die drei Teilungen Polens in den Jahren 1772, 1793 und 1795 und sein Verschwinden von der Landkarte für mehr als hundert Jahre hätten ihnen etwas anhaben können. (Nawrat 2015, S. 353)

Die Familie fungiert demnach als des Polen feste Burg und als die sozialste unter allen möglichen Gemeinschaften. Sie ersetzt aber auch - in der Darstellung Nawrats - die kaum im Land vorhandenen Strukturen einer modernen Zivilgesellschaft. Die sich in der Variabilität neuer Lebensmodelle widerspiegelnde Wandlung der posthistorischen Gesellschaften Westeuropas scheint im geschichtsfixierten Land aller Polen noch nicht richtig angekommen zu sein.

In diesem Kontext wäre eine von Wehleidigkeit eingefärbte Darstellung durchaus denkbar, Nawrat aber bietet eine flott lesbare, selbst für den auf oberflächliche Bildung durch Unterhaltung ausgerichteten Leser akzeptablere Alternative. Es ist ein Erzählmodus, der deutlich auf eine liebenswürdig bis sarkastisch dargebotene Situationskomik setzt. Nawrat fühlt sich eindeutig der erprobten Tradition eines stellenweise autobiographisch gefärbten Schelmenromans verpflichtet. Mit dieser Strategie ist er auch keineswegs ein Einzelfall. Schließlich bedienen sich auch andere Autoren deutscher Zunge mit polnischem Migrationshintergrund, etwa Radek Knapp (Knapp 2001) oder Artur Becker (Becker 1997), seit geraumer Zeit frei aus dem inzwischen recht prall gefüllten Depot allgemeinverständlicher literarischer Symbolik. Das Erbe von Till Eulenspiegel, Don Quichote, Peter Schlemihl, Josef Schwejk oder des Hrabalschen Onkel Pepin lebt und leibt in ihren Texten im Mikrokosmos der mitteleuropäischen Skurrilität fort. Es scheint gewissermaßen ein narratives Gegengift zu den in der polnischen Literatur - sei es als Programm, sei es als Antithese - überproportional präsenten Martyriums-Diskursen oder den romantisch inspirierten Erlösungsphantasien. Die Entscheidung der polnischstämmigen deutschen Schriftsteller, den für den deutschen Leser schwer zu entschlüsselnden Weichsel-Messianismus in ihren Polen-Texten nicht aufzugreifen, ist durchaus nachvollziehbar, da dieser Messianismus auf den - in der Ideen- und Geistesgeschichte Polens zumeist nur wenig bewanderten - deutschsprachigen Leser verwirrend wirken müsste, was den Unterhaltungsfaktor des Erzählten vermutlich senken würde.

Nawrats Geschichte beginnt im Warschau der Vorkriegszeit, das in Opa Jureks Erinnerung als eine bis in den Zustand einzigartiger Bilderbuchhaftigkeit hochidealisierte Stadt fortlebt, in der ,helle und große und dadurch besonders interessante Gebäude standen, mit Türmchen, Säulen und Erkern. Überhaupt ist damals in Warschau eine andere Zeit gewesen, viel kultivierter als heutzutage“. (Nawrat 2015, S. 13) Mit dem Ausbruch des Zweiten Weltkrieges beginnt die „sogenannte schwierige Zeit" (Nawrat 2015, S. 7) - es beginnen die breit angelegten deutschen Aktivitäten in Polen, deren Planmäßigkeit erschauern macht. Der märchenhaft-verwunschene Habitus einer heilen Vorkriegs-Wirklichkeit nimmt ein jähes Ende, als Opa Jurek mit dem Einmarsch der Deutschen konfrontiert wird. Diese seien „damals noch ganz 
anders [gewesen], als man sie heute kennt" (Nawrat 2015, S. 18), und allem Anschein nach wegen der 1410 in der Schlacht bei Grunwald/Tannenberg erlittenen, kläglichen Niederlage des Kreuzritterordens ,gegen den polnisch-litauischen Großkönig Władysław Jagiełło“ (Nawrat 2015, S. 18) aufs Äußerste „gekränkt in ihrem Stolz gewesen, weshalb sie nichts sehnlicher gewünscht hätten, als sich allumfassend zu rächen“. (Nawrat 2015, S. 18) Bald landet der Großvater in Auschwitz/ Oświęcim. Während seiner Auschwitz-Gefangenschaft tritt der Mann in die Fußstapfen des italienischen Juden Guido in Roberto Benignis Auschwitz-Tragikomödie La vita è bella. In „pyjamaartiger Arbeitsbekleidung“ (Nawrat 2015, S. 37) steckend, sieht er sich einer Welt gegenübergestellt, in der „eine Umkehrung aller Dinge“ (Nawrat 2015, S. 51) die pervertierte Norm zu sein scheint: „Denn in Wahrheit sei man in Oświęcim die ganze Zeit tot gewesen, obwohl man ja die ganze Zeit geglaubt habe, am Leben zu sein.“ (Nawrat 2015, S. 51)

„So gut wie kein historisches Gebäude, das wir heute in Opole kennen, war damals heil" (Nawrat 2015, S. 95): Das im Frühjahr des Jahres 1945 nahezu gänzlich zerstörte ehemalige Opeln - eine „Westernstadt auf dem Mond“ (Nawrat 2015, S. 88) - ersetzt dem Großvater nach dem Krieg das traute, wegen seiner restlosen Zerstörung umso heftiger verklärte Warschau der Kindheit und der Jugendzeit. Für die in die , wiedergewonnenen Gebiete' umgesiedelten Polen äußert sich die Rohheit der unmittelbaren Nachkriegsjahre zuallererst in dem Umstand, dass es für sie das Gebot der Stunde ist, sich, wenn auch zähneknirschend, so doch irgendwie mit den neuen Machthabern - den russischen Befreiern und Besatzern - zu arrangieren, jener „Geißel der Menschheit“ (Nawrat 2015, S. 89), die nach Belieben ihrem Hang zur Raubritterschaft frönen darf. Der Zustand permanenter physischer Bedrohung im rechtsfreien Raum dieser Stadt, der permanente Notstand einer desolaten Versorgung der Bevölkerung, schließlich das überwältigende Gefühl einer völlig unsicheren Zukunft - all das führt paradoxerweise dazu, dass sich selbst der seinerzeit aufs Schlimmste von den Nazis gepeinigte Großvater nach so mancher der deutschen Sekundärtugenden sehnt: ,und fast vermisste unser Opa bald die Verwaltungs- und Organisationskunst der Deutschen, denn denen wäre, wie er uns erklärt hat, ein solches Durcheinander nicht passiert". (Nawrat 2015, S. 92) Damit rekurrieren die WirErzähler Nawrats auf die in der polnischen Wahrnehmung des ,deutschen Wesense deutlich präsente Ambiguität: Auf der einen Seite ist es die Angst vor der Grausamkeit eines kaltblütig und exakt nach Plan wütenden Furor teutonicus, auf der anderen der Respekt vor den zivilisatorischen Leistungen und dem technologischen Vorsprung der Deutschen.

Bald nachdem sich die kommunistische Herrschaft in Polen etabliert hat - unter der Ägide Stalins, der spöttisch als „Freund des polnischen Volkes“ (Nawrat 2015, S. 135) bezeichnet wird -, beginnt die über vier Dezennien lang fortwährende Skurrilität des realsozialistischen Alltags mit ihren mal tragikomischen, mal schaurigen Facetten. Im Hintergrund der Erzählung sind alle Machthaber dieser Zeit vertreten - 
von dem Erzstalinisten Bolesław Bierut, über den Verfechter der behäbigen, polnischen Sozialismus-Variante Wladysław Gomułka und den gemäßigten Technokraten Edward Gierek bis hin zu dem Kriegsrechterklärer und unverhofften Totengräber des realexistierenden Sozialismus: Wojciech Jaruzelski. Opa Jurek darf den größten Teil dieser Zeit „als Mitglied der Großen Gemeinsamen Partei“ (Nawrat 2015, S. 169) erleben, was seiner Schelmenexistenz besondere Brisanz verleiht. Die Umtriebigkeit garantiert das Überleben, selbst wenn es sich um ein Überleben in einer gemessen an der BRD - Welt der zweiten Kategorie handeln sollte, die wenig Farbe und Frohsinn, dafür aber viel Mühe und Anlass zur Enthaltsamkeit bietet:

Alles ist angeblich grau gewesen, das behaupten unsere Eltern. Die Blicke der Nachbarn in den Fenstern: grau. Die Versammlung im Schulhof zum Appell: grau. Das Anstehen in der Verkaufsstelle für Geflügelfleisch [...], die Hähnchen, die kopfüber an den Haken an der Wand hingen: grau. (Nawrat 2015, S. 151)

Nach dem endgültigen Kollaps des realsozialistischen Systems konstatieren die Erzähler über die Epoche des Sozialismus, auf die Aktivitäten und Erinnerungen des Großvaters eingehend, Folgendes: „Im Grunde genommen habe es sich bei der Geschichte der letzten fünfzig Jahre, im Nachhinein betrachtet um eine Art weltweites Experiment gehandelt, bei dem die eine gegen die andere Überlegung angetreten sei.“ (Nawrat 2015, S. 380) Überhaupt scheint für Nawrat die Geschichte einen besonders gewichtigen Referenzrahmen für die Erzählung zu bilden. Herangezogen werden daher historische Gestalten, die dem Leser in Form eines Schnellkurses die Schlaglichter der polnischen Geschichte der letzten zwei Jahrhunderte näher bringen, u.a. Tadeusz Kościuszko, General Dąbrowski, Marschall Piłsudski, Johannes Paul II., Lech Wałęsa. Sie alle stehen gleichsam Pate für die Darstellung des historischen Abrisses und sind plakative Legitimierungselemente zugleich. Eine evidente Bestätigung, wenn nicht Verstärkung der gängigen Klischees findet im Bereich der jüngsten Vergangenheit statt: Mit Elan werden die eingestanzten Denkbilder des mit wahrhaft polnischer Verwegenheit geführten und völlig aussichtslosen Kampfes gegen eine fremde - in diesem Fall gegen die deutsche - Übermacht aufgefrischt. So manches symbolische Element des Ganzen stammt dabei noch aus der umtriebigen Werkstatt der Nazi-Propaganda. So etwa im Fall der Lüge, die polnische Kavallerie habe im September 1939 eine sinnlose Attacke gegen deutsche Panzer geführt; ein Bild freilich, das durch die Selbstdarstellung der Polen in Andrzej Wajdas Kriegsfilm Lotna in den Bereich der Eigensymbolik transponiert worden ist, um so den Stoff für zahlreiche Debatten über die Sinnlosigkeit der um jeden Preis erbrachten heroischen Gesten zu liefern.

Nawrat geht jedoch noch einen Schritt weiter: Bei der anschaulichen Aneinanderreihung von Bildern weicht die Diachronie der Erzählung einer Synchronie der dargestellten Ereignisse. Und so überliefert die kollektive Familien-Erinnerung der Wir-Erzähler, ein Großonkel sei angeblich ,in der schönsten polnischen Uniform 
der Heimatarmee AK mit bloßem Säbel gegen deutsche Panzer angeritten und [habe] den einen oder anderen Deutschen abgeschlachtet". (Nawrat 2015, S. 65) Die verwegenen Taten der regulären Ulanen-Kavallerie-Verbände im Polenfeldzug im September des Jahres 1939 verschmelzen so nahtlos mit der um einige Jahre und Erfahrungen später - genau genommen in den Jahren 1942-1945 - erfolgenden operativen Tätigkeit der während der deutschen Besatzung im Untergrund agierenden Heimatarmee AK. Auch ist die Gloriole der aus dem Londoner Exil heraus geführten Untergrundarmee selbst dann noch wahrnehmbar, als das Land an seiner Oberfläche längst schon realsozialistisch geworden zu sein scheint. Doch werden solche Geschichten nur inoffiziell im Zirkel der Eingeweihten weitererzählt. Noch in den frühen Sechzigern erlebt etwa der Vater der Erzählenden im Sommer-Pfadfinderlager regelmäßig Mittagsapelle, bei denen , in mehreren Partisanenliedern der Heimatarmee AK und der Exilregierung in Großbritannien unter Premierminister Stanisław Mikołajczyk während des Zweiten Weltkrieges gedacht wurde“. (Nawrat 2015, S. 71)

Neben den geschichtlichen Begebenheiten - und den innigen Verwandtschaftsbeziehungen - ist es auch die Kategorie der Sitten und Bräuche (in einem weit gefassten Sinne), die für Nawrat zum Darbietungsfeld des Polnisch-Seins erklärt werden. Der in einem Buch über Polen und die Polen wohl obligate Themenkomplex der ,polnischen Gastfreundschaft ${ }^{\star}$ ist ohne die Erwähnung diverser Delikatessen und Leibgerichte nicht denkbar. Stellenweise wird der Leser mit einem geradezu eruptiven kulinarischen Enthusiasmus konfrontiert. Schilderungen typisch polnischer Festmahlaktivitäten, Loblieder auf die deftige Hausmannskost sowie detaillierte Charakteristika einzelner Gerichte rücken in den Fokus der literarischen Darbietung. Ein Paradebeispiel dafür liefert etwa eine wahre Liebeserklärung an die kaszanka, „eine mit Kasza, also Buchweizengrieß, vermischte Blutwurst, [...] nichts Praktischeres auf der Welt gibt es als Kaszanka, eine komplette Mahlzeit in einer einzigen Darmhaut". (Nawrat 2015, S. 397) Die minutiöse Aufzählung der Lieblingsspeisen des verblichenen Großvaters umfasst „Schnitzel, Bigos, Pierogi, Rote-Bete-Suppe, Kuttelsuppe [...], aber auch Karpfen in Aspik. Und natürlich die berühmte Hasenpastete“. (Nawrat 2015, S. 397-398) Bereits nach der Bestattungszeremonie hegen die Erzähler die heiße Hoffnung, dass der Leichenschmaus ,aus Żurek bestehen werde oder wenigstens aus Kuttelsuppe, im schlimmsten Fall nur aus Pierogi“". (Nawrat 2015, S. 9-10) Pierogi seien ohnehin ,wahrscheinlich überhaupt das Beste, was von der Ukraine nach Polen gekommen ist [...], diese Art der Pierogi, die mit einer Kartoffel-Zwiebel-Quark-Füllung heute Russische Pierogi genannt wird“. (Nawrat 2015, S. 127) Zu einem anständigen Familientreffen in Polen gehören außerdem - „nach dem mehrgängigen Mittagessen“ (Nawrat 2015, S. 78) - „Kaffee mit Moccatorte am Sofatisch“ (Nawrat 2015, S. 60).

Der Standpunkt, von dem aus Opa Jureks Lebensgeschichte erzählt wird, zeichnet sich durch tiefe Empathie aus. Die Perspektive bewegt sich souverän zwischen den zwei Kulturen - jener der polnischen Herkunft und jener des Endgültig-in- 
Deutschland-Angekommen-Seins. Auf den ersten Blick haben sich die OpoleBesucher Nawrats längst schon aus der engen Bindung an das unmittelbare Alltagsleben des Herkunftslandes gelöst, wovon allein das Erstaunen über die Veränderungen zeugt, die sich in der Stadt und im Land vollzogen haben. Dieser Umstand wird bisweilen auch in unmissverständlichen Worten ausgedrückt: „Ihr habt eben inzwischen einen Akzent, sagt unser Onkel Wojtek." (Nawrat 2015, S. 61) Die Sicht der Erzählenden ist die Sicht der wohlwollenden Außenstehenden, die einen dank der Entfernung geschärften Blick auf die Besonderheiten richten. Dieses mehrstimmige Erzählmedium hat keineswegs die Absicht, die eigenen Wurzeln und die eigene Herkunft zu verleugnen, dafür ist das aus guten Erinnerungen geknüpfte Band unversehrter Familienbeziehungen viel zu fest gezogen. Auch gab es zu dem Zeitpunkt, als es am Ausgang des Kindesalters und unmittelbar vor dem Übergang in die Phase der Adoleszenz galt, nach der folgenschweren Entscheidung der eigenen Eltern, mit ihnen die alte Heimat zu verlassen und in eines der Länder ,auf der anderen Seite der DDR“ (Nawrat 2015, S. 172) auszuwandern, bereits zu viele Sozialisierungsschwerpunkte. Die primäre Identität der erzählenden Instanz war schließlich die polnische. Zudem wurden die Auswanderer kurz vor ihrem Umzug nach Deutschland seinerzeit von Opa Jurek streng ermahnt, „dass [sie] gefälligst nicht vergessen sollten, woher [sie] kämen. [Sie] sollten nicht vollständig zu Deutschen werden, obwohl die Deutschen ja auch normale Menschen seien, zumindest heute.“ (Nawrat 2015, S. 84)

Die Ereignisse von einst sind jederzeit aus dem Gedächtnis abrufbar. Sie wirken bis in den heutigen Tag hinein in Form von nostalgischen Rückblenden und mit Hilfe einer sich geradezu aufzwingenden Vergleiches mit dem Heute:

\footnotetext{
Viele Dinge gehen in unserer Siedlung vor, das haben wir schon bei unseren letzten Besuchen in Opole festgestellt. [...] Dafür ist die Metzgerei im Block 26 nicht mehr da, und im ehemaligen Supermarktgebäude hinter dem Block 13 befinden sich jetzt ein Computergeschäft, eine Pizzeria und eine Apotheke, denn es gibt inzwischen einen noch größeren Supermarkt auf der anderen Seite der Siedlung. (Nawrat 2015, S. 149)
}

Während dieser Streifzüge durch das heutige Opole werden unvermittelt die Erinnerungen der erzählenden Instanz an die eigene Kindheit wachgerufen. Die zahlreichen Besuche bei den lange nicht gesehenen Angehörigen verstärken noch die Empfindung der trauernden Enkelkinder, dass sich die aktuelle Realität unvermittelt in die Sphäre des längst als Vergangen geglaubten verschieben kann.

Die sekundäre Sozialisierung erfolgte für die Wir-Erzähler Ende der 1980er Jahre im festen Gefüge der als Wohlstands- und Ordnungsdestination empfundenen Bundesrepublik Deutschland. Dennoch ist Deutschland nicht etwa die alleinige geistige Heimat der Erzählenden. Die Übergänge zwischen den beiden gesellschaftlichen wie mentalen Sphären des deutschen und des polnischen Selbstverständnisses sind nach wie vor fließend. Die schillernde Wir-Instanz selbst begreift sich als 
Medium einer intimen Kenntnis der polnischen Seele und als selbsternannte Kapazität auf dem Gebiet ihrer - der polnischen - Geschichte. Ihre Aufgabe sieht sie darin, dem Leser pflichtbewusst das aus ihrer Sicht Bedeutsame über das Land und seine Leute zu vermitteln: „Und wir denken, dass es schön ist, zur Beerdigung unseres Opas Jurek in Opole gewesen zu sein und uns an alles erinnert zu haben." (Nawrat 2015, S. 406)

\section{Bibliographie}

Becker, A. (1997). Der Dadajsee. Roman. Bremen: Stint Verlag.

Bleutge, N. (13. Oktober 2015). Der Yukon unten im Keller. Süddeutsche Zeitung. Abgerufen von http://www.sueddeutsche.de

Halter, M. (12. April 2012). Matthias Nawrat: „Wir zwei allein“: Einsam, zweisam, Dreisam. Frankfurter Allgemeine Zeitung. Abgerufen von http://www.faz.net

Hayer, B. (10. März 2014). Matthias Nawrats Roman. Die Familie als Kapitalgesellschaft. Die Zeit. Abgerufen von http://www.zeit.de

Knapp, R. (2001). Herrn Kukas Empfehlungen. Roman. München: Piper.

Kurianowicz, T. (15. Oktober 2015). Kopf eines Königs, Körper eines Hofnarrs. Matthias Nawrat erzählt ein polnisches Jahrhundertleben als Schelmenroman. Frankfurter Allgemeine Zeitung. Abgerufen von http://www.faz.net

Mihułka, K. (2010). Stereotype und Vorurteile in der deutsch-polnischen Wahrnehmung. Eine empirische Studie zur Evaluation des Landesbildes durch Germanistikstudenten. Rzeszów: Wydawnictwo Uniwersytetu Rzeszowskiego.

Nawrat, M. (2015). Die vielen Tode unseres Opas Jurek. Roman. Reinbek bei Hamburg: Rowohlt.

Orłowski, H. (1996). ,Polnische Wirtschaft: Zum deutschen Polendiskurs der Neuzeit. Wiesbaden: Harrassowitz.

Orłowski, H. (2005). Die Lesbarkeit von Stereotypen. Der deutsche Polendiskurs im Blick historischer Stereotypenforschung und historischer Semantik. Wrocław: Neisse Verlag.

Pająk-Patkowska, B. (2011). Kilka słów wstępu. In: Dies. (Hrsg.), Stereotypy w obszarze społecznym i politycznym. Poznań: Wydawnictwo Naukowe WNPiD UAM, S. 5-6.

Popovic, B. (1998). Stereotype im Fremdsprachenunterricht in einer multikulturellen Gesellschaft (S. 205-224). In M. Löschmann \& M. Stroinska (Hrsg.), Stereotype im Fremdsprachenunterricht. Frankfurt a.M.: Peter Lang.

Végel, L. (2008). Mitteleuropäische Alchimie. Aus dem Ungarischen von Laszlo Kornitzer. Literatur und Kritik, 423/424, 48-55. 